\title{
Discussing the psychiatric manifestations of 22q11.2 deletion syndrome: an exploration of clinical practice among medical geneticists
}

\author{
Emily Morris, $\mathrm{MSc}^{1}$, Angela Inglis, MSc ${ }^{1,2}$, Jan Friedman, MD, $\mathrm{PhD}^{1}$ and Jehannine Austin, $\mathrm{PhD}^{1,2}$
}

\begin{abstract}
Purpose: The aim of this study was to determine the frequency with which medical geneticists discuss the psychiatric manifestations of 22q11.2 deletion syndrome (22q11DS) with families in relation to the frequency with which they discuss the other manifestations of the syndromes and to explore relationships between discussion of these features and stigma toward psychiatric disorders.
\end{abstract}

Methods: We surveyed medical geneticists in the United States and Canada regarding the frequency with which they discuss various features of 22q11DS with families in the context of four clinical scenarios in which only the age of the patient at diagnosis differed. Respondents also completed a 20 -item validated psychometric measure of stigma toward psychiatric disorders.

Results: 308 of 546 medical geneticists completed the survey (56\% response rate). Overall, psychiatric disorders were discussed signifi-

\section{INTRODUCTION}

The 22q11.2 deletion syndrome (22q11DS) is the most common microdeletion syndrome, affecting 1 of 4,000 newborns. ${ }^{1}$ Individuals with 22q11DS present with a range of clinical manifestations, with congenital cardiac and palate defects, calcium deficiencies, immune problems, and learning and cognitive disabilities among the most common. ${ }^{2,3}$ In addition, individuals with 22q11DS seem to have elevated rates of attention-deficit hyperactivity disorder, anxiety, oppositional defiant disorder, and autism spectrums disorders, and approximately $25-30 \%$ of individuals with 22q11DS develop schizophrenia or other psychotic disorders. ${ }^{3-13}$

In a 2008 study, parents of individuals with 22q11DS $(n=41)$ reported that psychiatric disorders were their second most important cause for concern related to the syndrome (after learning disabilities) and that of all the manifestations of the condition, psychiatric disorders were the source of their greatest anxiety. ${ }^{14}$ In addition, and crucially, many parents reported that they did not receive information about the psychiatric risks associated with 22q11DS from their health-care provider but instead obtained information about these risks from the Internet. $^{14}$

The group of physicians who are most often involved in delivering a diagnosis of 22q11DS are medical geneticists. ${ }^{14}$ Although a recent exploratory descriptive study examined the attitudes of cantly less often than other features of 22q11DS $(P<0.0001)$ but were discussed significantly more often when the patient was 13 years or older $(P<0.0001)$ than when the patient was younger. Geneticists who discussed psychiatric disorders the least had significantly higher levels of stigma toward psychiatric disorders $(P=0.007)$.

Conclusion: Psychiatric manifestations of 22q11DS are less often discussed with families during childhood. Education for physicians to help reduce stigma toward psychiatric disorders (which may impede discussion of psychiatric disorders) may warrant exploration in this population.

Genet Med advance online publication 11 April 2013

Key Words: 22q11.2 deletion syndrome; medical geneticists; mental illness; psychiatric disorders; stigma

54 genetic counselors toward providing information about psychiatric risks in the context of 22q11DS, ${ }^{15}$ there are no reported studies assessing the practices of medical geneticists in relation to discussing psychiatric manifestations of the condition.

Understanding how medical geneticists approach discussing the features of 22q11DS - and in particular, the psychiatric features of this syndrome-is both important and timely, given (i) the recent publication of international practice guidelines ${ }^{16}$ that recommend that psychiatric symptoms should be assessed repeatedly in children with 22q11DS from preschool through adolescence, (ii) a recent report stating that mental health resources are underutilized by patients with 22q11DS ${ }^{17}$ (which suggests that psychiatric disorders may be undiagnosed in this population), and (iii) the current movement toward including 22q11DS in some newborn screening programs. ${ }^{18,19}$

It is relevant to note that psychiatric disorders are among the most profoundly stigmatized of all health conditions, and negative attitudes toward these conditions have been identified in many groups including the general public, ${ }^{20-23}$ students, ${ }^{24}$ police, ${ }^{25}$ mental health workers, ${ }^{26,27}$ and genetic counselors. ${ }^{28,29}$ Medical geneticists' attitudes toward individuals with psychiatric disorders, and the relationships between these attitudes and clinical practice regarding disclosure of information about psychiatric risks, have not been previously studied. It is possible, however, that medical geneticists who have more negative 
attitudes toward people with psychiatric disorders may be less likely to discuss psychiatric risks with families and individuals with 22q11DS.

We designed a cross-sectional survey-based study to test the hypotheses that (i) overall, medical geneticists discuss psychiatric manifestations of 22q11DS less often than five other common features of the syndrome; (ii) medical geneticists discuss the psychiatric disorders less frequently with families whose affected child is 12 years or younger, and more frequently with families when the affected child is 13 years or older; and (iii) medical geneticists who have more stigmatizing attitudes toward psychiatric disorders discuss psychiatric manifestations with families less often than medical geneticists who have less stigmatizing attitudes toward psychiatric disorders.

\section{MATERIALS AND METHODS}

We obtained institutional review board approval for the study from the University of British Columbia (H11-00689). We obtained contact information for clinical geneticists through the membership directories of the Canadian College of Medical Geneticists and the American College of Medical Genetics. To each North American medical geneticist, we sent a survey package, including a consent form, the survey (described below), a stamped addressed return envelope, a pencil, and a $\$ 5.00$ Starbucks gift card. ${ }^{30}$ Concurrently with the survey mailout, e-mails were sent to inform potential participants about the study and to alert them to look out for the survey package in the mail. All respondents were given the option of completing the survey online (via http://www.qualtrics.com) or completing the hardcopy and returning it by mail. Reminder e-mails were sent approximately 4 and 6 weeks after the initial mailout. Survey responses were collected between October 2011 and February 2012.

The survey included demographic items (gender, age, years in clinical practice, number of patients with 22q11DS seen per year, and personal experience with psychiatric disorders; Table 1).

Respondents also reported the frequency (always, often, sometimes, rarely, or never) with which they discuss six common features of 22q11DS (cardiac defects, immune problems, low serum calcium concentration, palate abnormalities, learning disabilities, and psychiatric disorders) with families in four different scenarios: S1, when the affected individual is diagnosed prenatally or within the first year after birth; S2, during childhood (1-12 years of age); S3, during adolescence (13-18 years of age); and S4, in adulthood (19 years or older).

Medical geneticists who indicated that they "rarely" or "never" discuss psychiatric disorders for any of the scenarios were prompted to provide a rationale for their approach. We provided a list of six possible options (Table 2). We also allowed respondents to indicate "other" and write in their own alternative answer.

The survey also included the Opening Minds Scale for Health Care Providers (OMS-HC) stigma scale, a 20-item validated measure that assesses health-care providers'
Table 1 Demographic information of medical geneticists $(n=308)$

\begin{tabular}{|c|c|c|}
\hline Characteristic & $n$ & $\%$ \\
\hline \multicolumn{3}{|l|}{ Gender $^{a}$} \\
\hline Male & 141 & 46.69 \\
\hline Female & 161 & 53.31 \\
\hline \multicolumn{3}{|l|}{$\operatorname{Age}^{b}$} \\
\hline$<30$ years & 0 & 0.00 \\
\hline $31-40$ years & 36 & 11.84 \\
\hline $41-50$ years & 74 & 24.34 \\
\hline $51-60$ years & 122 & 40.13 \\
\hline$>61$ & 72 & 23.68 \\
\hline \multicolumn{3}{|l|}{ Location of clinical practice ${ }^{c}$} \\
\hline Other & 1 & 0.33 \\
\hline Canada & 48 & 15.89 \\
\hline United States & 253 & 83.77 \\
\hline \multicolumn{3}{|l|}{ Have you ever had a patient with 22q11DS?d } \\
\hline Yes & 295 & $96.40 \%$ \\
\hline No & 11 & $3.6 \%$ \\
\hline \multicolumn{3}{|l|}{ Number of cases of 22q11DS per yeare } \\
\hline $0-1$ cases & 64 & 22.07 \\
\hline $2-3$ cases & 93 & 32.07 \\
\hline 4-6 cases & 69 & 23.79 \\
\hline 7-9 cases & 21 & 7.24 \\
\hline$>10$ cases & 43 & 14.83 \\
\hline \multicolumn{3}{|l|}{ Personal experiences with mental illness } \\
\hline I have a mental illness & 14 & 3.23 \\
\hline $\begin{array}{l}\text { I have one or more family member(s) with } \\
\text { mental illness }\end{array}$ & 141 & 32.49 \\
\hline I have a close friend who has a mental illness & 68 & 15.67 \\
\hline I have a work colleague with a mental illness & 69 & 15.90 \\
\hline $\begin{array}{l}\text { I have worked/volunteered with a mental } \\
\text { health organization }\end{array}$ & 28 & 6.45 \\
\hline $\begin{array}{l}\text { Other (respondents could elaborate on } \\
\text { experience) }\end{array}$ & 44 & 10.14 \\
\hline $\begin{array}{l}\text { I do not have any acquaintances with a } \\
\text { mental illness }\end{array}$ & 70 & 16.13 \\
\hline
\end{tabular}

Respondents could select more than one option for "personal experiences with mental illness." Total numbers may not add up to 308 because of missing data points, given that some respondents chose not to answer certain demographic characteristics.

22q11DS, 22q11.2 deletion syndrome.

asix individuals did not provide their gender. ${ }^{b}$ Four individuals did not provide their age. 'Six individuals did not provide their location of clinical practice. 'Two individuals did not indicate whether they have ever had a patient with 22q11DS. eFive individuals who indicated that they have seen patients with 22q11DS did not indicate how many cases of 22q11DS they see per year.

stigmatizing attitudes toward psychiatric disorders. ${ }^{31}$ The OMS-HC stigma scale has five content areas: social distance, discrimination and devaluation, help seeking and disclosure, recovering, and social responsibility. Each item on the OMS-HC stigma scale is rated on a five-point anchored Likert 
Table 2 Rationale provided by medical geneticists who indicated rarely or never discussing psychiatric disorders during diagnosis of 22q11DS in which diagnosis was made at S1 (prenatal to $<1$ year) or S2 (1-12 years) $(n=38)$

\begin{tabular}{lccc} 
Rationale & $\boldsymbol{n}$ & $\%$ \\
\hline $\begin{array}{l}\text { The chance of psychiatric disorders in patients with } \\
\text { 22q11DS is very low }\end{array}$ & 4 & 7 \\
\hline $\begin{array}{l}\text { I do not want to worry parents about psychiatric disorders } \\
\text { Psychiatric disorders are not a significant issue; other }\end{array}$ & 5 & 8 \\
$\begin{array}{l}\text { conditions are more important } \\
\text { Discussing the risks for psychiatric disorders is the }\end{array}$ & 1 & 2 \\
responsibility of another professional (e.g., psychiatrist) & & \\
$\begin{array}{l}\text { Psychiatric disorders are not a relevant issue for patients } \\
\text { with 22q11DS at this age }\end{array}$ & 17 & 28 \\
$\begin{array}{l}\text { I would discuss risks for psychiatric disorders at a follow- } \\
\text { up appointment when the child is older }\end{array}$ & 23 & 38 \\
\hline Other & 8 & 13 \\
\hline
\end{tabular}

Respondents who indicated "other" were able to provide their own rationale. Respondents were able to check more than one response, if they desired.

22q11DS, 22q11.2 deletion syndrome.

scale (1: strongly disagree and 5: strongly agree); scale scores are derived by summing item scores and dividing by the number of items answered, with higher scores indicating greater stigma toward psychiatric disorders. Respondents were given the opportunity to provide additional comments about any of the survey questions.

\section{Data analyses}

We excluded survey packages that were returned to sender as a result of invalid delivery address from our total number for our response rate calculation. Medical geneticists who indicated that they had never seen patients on a clinical basis or had only seen patients in a nonrelevant subspecialty (e.g., cancer) were also excluded. We considered a survey complete only if the respondent had filled out one or more of the four scenarios about their clinical practice related to discussion of various manifestations of 22q11DS, or had completed 15 or more of the OMS-HC stigma scale items (we used mean item scores rather than total scores in analyzing data from the OMS-HC stigma scale), and did not exclude surveys returned with incomplete demographic information. Descriptive statistics were calculated for demographic data. To determine whether psychiatric disorders were discussed less frequently than other manifestations of 22q11DS (hypothesis 1), we generated composite "frequency of discussion" scores for each of the six manifestations of 22q11DS included in the questionnaire by scoring "always" as 5 and "never" as 1 and combining scores from all four scenarios. We then used repeated-measures analysis of variance to compare the frequency of discussion scores for the six manifestations of 22q11DS. In addition, we generated an overall frequency of discussion score for all the nonpsychiatric manifestations combined and performed a $t$-test to compare the frequency of discussion scores between psychiatric disorders and nonpsychiatric manifestations.
To compare the frequency with which psychiatric disorders are discussed when the patient is an infant or child in relation to when the patient is an adolescent or adult, we used a $t$-test to compare two mean frequency of discussion scores for psychiatric disorders. The first we produced by combining data from $\mathrm{S} 1$ and S2. The second we produced by combining data from S3 and S4.

To determine whether respondents with more stigmatizing attitudes toward psychiatric disorders discuss those disorders less with families with 22q11DS, we used multivariate analysis of variance to compare the OMS-HC stigma scale scores and scores for each of the five content areas of the OMS-HC stigma scale between those who reported that they "rarely or never" and those who "often or always" discuss psychiatric disorders.

In an exploratory analysis, we also used multivariate analysis of variance to compare the frequency of discussion of psychiatric risks and OMS-HC stigma scale scores between respondents grouped on the basis of the number of patients with 22q11DS seen per year.

For all tests, a significance threshold $(\alpha)$ of $P<0.01$ was applied (to allow for the above five tests at a nominal overall significance level of 0.05 ). We calculated internal consistency of the OMS-HC stigma scale for our population of medical geneticists using Cronbach's $\alpha$.

\section{RESULTS}

Of 597 surveys sent out, 51 were excluded from the analyses as a result of ineligibility (as described in the Materials and Methods) or having an incorrect address, and 308 completed surveys were returned $(597-51=546 ; 308 / 546=56 \%)$. Most respondents completed the questionnaire by mail $(n=231)$, with the remainder $(n=77)$ completing it online. Demographic information and information about participants' clinical experience with 22q11DS are displayed in Table 1. All participants were medical geneticists who had provided clinical service at some point in their career, and $92 \%(n=281)$ were currently practicing clinically, with a mean number of years in clinical practice of 19.8 years. A total of 105 respondents provided comments in the optional open-ended question at the end of the survey.

\section{Discussion of psychiatric vs. other manifestations during counseling following diagnosis of 22q11DS}

Repeated-measures analysis of variance with a GreenhouseGeisser correction showed that the overall mean frequency of discussion scores for the six manifestations of 22q11DS were statistically significantly different, $F(3.417,319.653)=35.820$ $(P<0.0001)$. Psychiatric disorders were discussed significantly less often than cardiac defects $(P<0.0001)$, palate defects $(P=$ $0.004)$, or learning disabilities $(P<0.0001)$. There was no significant difference between frequency of discussion of psychiatric disorders and frequency of discussion calcium deficiencies or immune problems. Supplementary Table S1 online shows the breakdown of the number of respondents who indicated 
"rarely," "never," "sometimes," "often," or "always" discuss psychiatric disorders for each of the four scenarios.

Comparing the pooled frequency of discussion scores for all nonpsychiatric manifestations with the frequency of discussion scores for psychiatric disorders revealed that the psychiatric manifestations of 22q11DS were discussed significantly less often than the other, nonpsychiatric features of the condition $t(287)=-3.87(P<0.0001)$. However, psychiatric disorders were discussed significantly more often when the patient was 13 years or older as compared with when the patient was 12 years or younger, $t(258)=-5.578(P \leq 0.0001$; Figure 1$)$. The rationales for not discussing psychiatric manifestations of 22q11DS when the diagnosis was made in S1 and S2 are shown in Table 2.

\section{Stigmatizing attitudes toward mental illness and discussion of psychiatric risks}

Table 3 shows the data from the OMS-HC stigma scale overall $(n=305)$ as well as its five content areas in this population of medical geneticists. Three respondents' OMS-HC stigma scale data were excluded for being incomplete (i.e., $<15$ items answered). Internal consistency of the OMS-HC stigma scale in this population was good (Cronbach's $\alpha=0.77$ ). There were significant differences in OMS-HC stigma scale scores between medical geneticists who reported "rarely" or "never" discussing psychiatric disorders for at least one of the age groups $(n=38)$ and those who "often" or "always" discussed psychiatric disorders, $F(12,590)=1.857(P=0.028$; Wilk's $\lambda=0.917)$. Table 4 shows further characteristics of these respondents. More specifically, respondents who "rarely" or "never" discussed psychiatric disorders had significantly different scores for both total OMS-HC, $F(2,301)=4.418(P=0.013)$ and the social responsibility content area, $F(2,301)=6.399(P=0.002)$.
Post hoc Tukey's test showed that both OMS-HC stigma scale total score and the social responsibility content area score were significantly higher for respondents who "rarely" or "never" discuss psychiatric disorders $(P=0.007$ and $P=0.001$, respectively). Scores in the discrimination and devaluation content area showed a trend $(P=0.016)$ toward higher scores among respondents who "rarely" or "never" discuss psychiatric disorders.

\section{Exploratory analyses}

There were no significant differences in OMS-HC stigma scale scores between respondents based on the number of cases of 22q11DS seen per year. There was, however, a significant difference in the frequency of discussion of psychiatric disorders when the total sample was stratified by number of cases of 22q11DS seen per year, $F(5,282)=5.487(P<0.0001)$. Tukey's post hoc comparisons showed that respondents who reported that they saw $\geq 10$ patients with 22q11DS per year discussed psychiatric disorders significantly more often than respondents who reported that they saw $0-1$ cases per year $(P<0.0001)$.

\section{DISCUSSION}

This study was the first to explore medical geneticists' approaches to discussing features of 22q11DS with families at the time of counseling following diagnosis, and the first to explore stigmatizing attitudes toward psychiatric disorders among medical geneticists. We found that the psychiatric manifestations of 22q11DS were discussed with families less often than the other features of 22q11DS overall (when all categories of age of patient were pooled). However, subsequent analyses supported our hypothesis by showing that the frequency with which psychiatric disorders were discussed was highly dependent on the age of the patient at diagnosis of 22q11DS-psychiatric

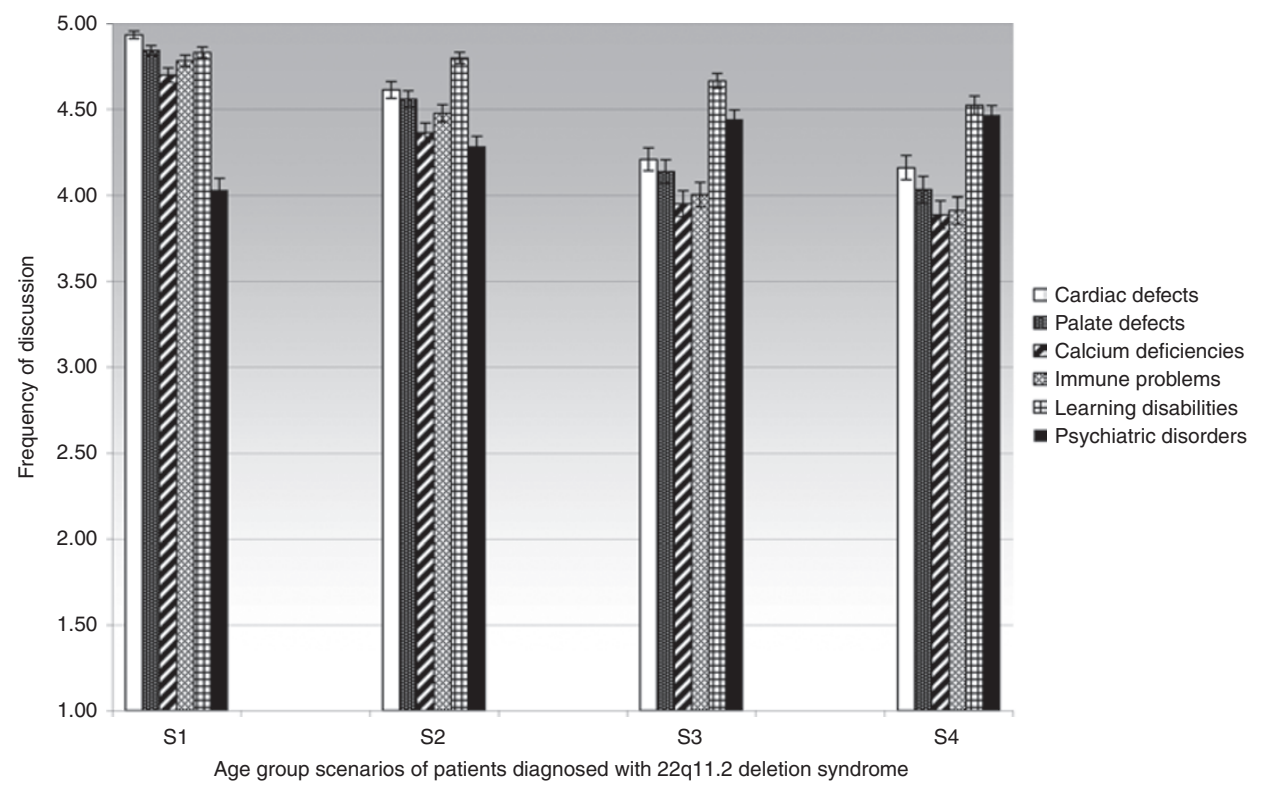

Figure 1 Breakdown of the mean frequency of discussion scores for each of the six main features of 22q11DS (cardiac defects, palate defects, calcium deficiencies, immune problems, learning disabilities, and psychiatric disorders) at S1 (prenatal to <1 year), S2 (1-12 years), S3 (13-18 years), and S4 ( $\geq 19$ years). Standard Error Mean error bars are shown. 
Table 3 Descriptive statistics from the OMS-HC stigma scale $(n=305)$

\begin{tabular}{lcccc} 
& Minimum & Maximum & Mean & SD \\
\hline Total mean item score & 1.2 & 3.3 & 2.3 & 0.368 \\
$\begin{array}{l}\text { Social distance mean item } \\
\text { score }\end{array}$ & 1.0 & 3.8 & 2.2 & 0.514 \\
$\begin{array}{l}\text { Discrimination and } \\
\text { devaluation mean item } \\
\text { score }\end{array}$ & 1.1 & 4.0 & 2.3 & 0.489 \\
$\begin{array}{l}\text { Help seeking mean item } \\
\text { score }\end{array}$ & 1.0 & 4.5 & 3.0 & 0.606 \\
$\begin{array}{l}\text { Recovering mean item } \\
\text { score }\end{array}$ & 1.0 & 5.0 & 2.9 & 0.979 \\
$\begin{array}{l}\text { Social responsibility mean } \\
\text { item score }\end{array}$ & 1.0 & 3.3 & 1.9 & 0.766
\end{tabular}

item score

A total of 283 respondents completed all 20 items; the remaining 22 respondents answered 15 or more of the total 20 items.

OMS-HC, Opening Minds Scale for Health Care Providers.

Table 4 Demographic characteristics of those respondents who indicated they "rarely" or "never" discuss psychiatric disorders during diagnosis of 22q11DS for at least one of the clinical scenarios (S1, S2, S3, or S4) $(n=38)$

\begin{tabular}{|c|c|c|}
\hline Characteristic & $n$ & $\%$ \\
\hline \multicolumn{3}{|l|}{ Gender } \\
\hline Male & 19 & 50.0 \\
\hline Female & 19 & 50.0 \\
\hline \multicolumn{3}{|l|}{$\mathrm{Age}^{\mathrm{a}}$} \\
\hline $31-40$ & 4 & 11.1 \\
\hline $41-50$ & 13 & 36.1 \\
\hline $51-60$ & 9 & 25.0 \\
\hline$>61$ & 10 & 27.8 \\
\hline \multicolumn{3}{|c|}{ Currently in clinical practice } \\
\hline Yes & 36 & 94.7 \\
\hline No & 2 & 5.3 \\
\hline \multicolumn{3}{|c|}{ Location of clinical practice } \\
\hline Canada & 4 & 10.5 \\
\hline United States & 34 & 89.5 \\
\hline \multicolumn{3}{|c|}{ Number of cases of 22q11DS seen per yearb } \\
\hline $0-1$ & 12 & 32.4 \\
\hline $2-3$ & 11 & 29.7 \\
\hline $4-6$ & 11 & 29.7 \\
\hline $7-9$ & 1 & 2.7 \\
\hline$>10$ & 2 & 5.4 \\
\hline
\end{tabular}

22q11DS, 22q11.2 deletion syndrome.

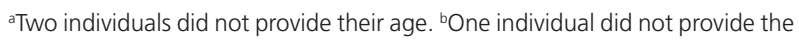
number of cases per year.

disorders were significantly less likely to be discussed when the diagnosis of 22q11DS was being made in childhood and significantly more likely to be discussed when the affected individual was an adolescent or adult. Our formulation of this hypothesis arose as a result of our perception that the bulk of the literature concerning psychiatric disorders and 22q11DS focused on psychotic disorders (and in particular schizophrenia), which tend to emerge during adolescence/young adulthood. Indeed, the rationales most frequently provided by respondents in this study for not discussing psychiatric disorders were: "Psychiatric disorders are not a relevant issue for patients with 22q11DS at this age," and "I would discuss risks for psychiatric disorders at a follow-up appointment when the child is older." These data are congruent with the findings of a recent descriptive study by Martin et al. ${ }^{15}$ which showed that genetic counselors were less likely to discuss psychiatric disorders than other features of 22q11DS during diagnoses in infancy because these issues would be addressed in follow-up visits.

This rationale indicates that perhaps there are some gaps in awareness among medical geneticists regarding risks for childhood-onset psychiatric conditions associated with $22 \mathrm{q}_{11 \mathrm{DS}^{7,12,13}}$ and suggests that perhaps respondents were focusing primarily on psychotic illnesses when responding to this question. Indeed, some comments spontaneously volunteered by participants indicate a potential focus on psychotic disorders. For example:

"Parents want to know that their child is going to be ok NOW before they want details about things that MIGHT happen in 21 years."

Regardless, however, parents of individuals with 22q11DS have indicated that they would prefer to receive information about potential psychiatric symptoms before the age of onset (i.e., when the patient is younger) ${ }^{15}$. In addition, this approach necessitates another clinic visit when the child is older in order for a family to find out about psychiatric manifestations of the illness from their medical geneticist. However, parents have reported that they were not told about the psychiatric manifestations of 22q11DS by their health-care providers. ${ }^{14}$ Furthermore, studies show significant underutilization of mental health resources (both pharmaceutical and nonpharmaceutical) by individuals with 22q11DS (perhaps reflecting underrecognition of psychiatric manifestations of the condition). ${ }^{17}$ Taken together, these findings suggest a number of possibilities: first, perhaps these follow-up visits are happening, and parents are, in fact, being told about the psychiatric disorders associated with the condition but fail to remember (perhaps as a result of information overload); second, perhaps follow-up visits are happening, but psychiatric disorders are not discussed; third, perhaps these follow-up visits with medical geneticists-at which psychiatric disorders would be discussed-may not routinely be occurring. Indeed, one respondent volunteered:

\section{“... These children don't come back to genetics typically."}

If these patients are in fact often not seen for follow-up visits with medical geneticists (perhaps even despite best intentions on the part of the clinician), this could be a crucial factor in determining whether families learn about psychiatric manifestations of 22q11DS from a health-care professional; it suggests 
that although current guidelines recommend that genetic counseling for patients with 22q11DS should be repeated at each life stage, especially during the transitional years from childhood to adulthood, ${ }^{16,17}$ this may not be happening in practice. This represents an important area for future research, especially given the current discussion regarding whether 22q11DS is a condition that should potentially be included in newborn screening panels. ${ }^{18,32}$ If $22 \mathrm{q} 11 \mathrm{DS}$ is included in newborn screening, diagnoses will increasingly be made at earlier ages. Because followup visits may not be occurring as often as intended, it will be important to identify strategies to facilitate return visits for patients and their families.

Furthermore, recent international practice guidelines for the management of patients with 22q11DS recommend repeated psychiatric assessments beginning in early childhood ${ }^{16}$ because early intervention and effective treatments greatly improve prognosis and reduce morbidity. ${ }^{33-35}$ However, if psychiatric manifestations of the syndrome are not discussed during childhood, appropriate psychiatric care and assessments are unlikely to occur. Therefore, an argument could be made that despite the fact that there is a lot of information to convey to parents when a diagnosis of 22q11DS is made in a child, it may be appropriate to include psychiatric manifestations of the condition in the discussion. It is certainly true that medical geneticists are not the only physician group involved in providing care for these patients. Indeed, pediatricians and/or family physicians are better placed to provide long-term ongoing care but may lack familiarity with the various manifestations of rare genetic conditions-this remains the specialist domain of the medical geneticist. ${ }^{17}$

\section{Discussion of psychiatric risks: an issue of stigma?}

Our results show that the frequency with which medical geneticists discuss psychiatric manifestations of 22q11DS is related to stigma: those who did not discuss this issue had significantly more stigmatizing attitudes toward mental illness overall. On closer inspection, this significant result was driven by differences in the social responsibility content area. The three items in this content area are thematically the most closely linked to attitudes toward providing information and support to patients about psychiatric disorders. For example, one item in the social responsibility content area is "Healthcare providers do not need to be advocates for people with mental illness": intuitively it seems consistent that those who agree with this statement may be less likely to provide information about psychiatric disorders in the context of 22q11DS. Our findings, which link health-care professionals' own stigmatizing attitudes toward psychiatric disorders with their approach to disclosing risks for psychiatric disorders are congruent with, yet expand upon, the findings of recent work. A recent study showed that genetic counselors were hesitant to disclose the risk for psychiatric disorders to families due, in part, to concerns about societal perceptions of mental illness and the impact on how parents may treat the affected child. The influence of health-care professionals' own stigma toward psychiatric disorders on clinical practice, however, was not explicitly explored. ${ }^{15}$
Many respondents provided comments in the designated space at the end of the survey, some of which provide further insight into respondents' attitudes toward mental illness. Some of these quotes may be interpreted as illustrating a more negative attitude toward certain kinds of psychiatric disorders (e.g., schizophrenia) than others (e.g., anxiety or depression)-a phenomenon that has been observed in studies in other populations. ${ }^{36,37}$ For example:

"Other illnesses (e.g. anxiety and depression) are more easily seen as compassion-worthy. Still others (schizophrenia and bipolar disorder) may best be served by being classed as neurological/neurodegenerative disorders."

"A person who takes Prozac to improve mood is not the same as a schizophrenic with a record of frequent drug holidays."

The OMS-HC stigma scale, however, does not distinguish between different psychiatric diagnoses. This suggests that although we clearly showed that those who rarely or never discuss psychiatric disorders in clinical practice had higher levels of stigmatizing attitudes toward psychiatric disorders (broadly defined), both overall and in the social responsibility content area, had the instrument been targeted specifically at a psychiatric disorder such as schizophrenia, we may also have found significant differences in additional content areas. This may constitute a fruitful area for future investigation.

Although psychiatric disorders were discussed significantly less often than other manifestations of 22q11DS overall, and when diagnosis was established prenatally or during childhood in particular, it is interesting to note that only 38 respondents indicated that they rarely or never discuss psychiatric risks with families. These results suggest that even when diagnoses of 22q11DS are made during childhood, most medical geneticists discuss psychiatric disorders at least some of the time. It is important to consider how these data can be reconciled with the findings of previous work that showed that parents reported not receiving information about the psychiatric manifestations of 22q11DS from their health-care providers. ${ }^{14}$ First, in addition to the medical geneticists who indicated "rarely" or "never" discussing psychiatric disorders, many others (see Supplementary Table S1) indicated "sometimes" or "often," rather than "always." Therefore, altogether, there is likely to be a substantial number of patients who receive a diagnosis of 22q11DS in which psychiatric risks are not discussed, and, as a result, many parents are indeed uninformed of these risks. Second, perhaps for the most part medical geneticists do in fact usually tell parents about psychiatric manifestations of 22q11DS, but due to the overwhelming amount of information they receive, parents do not recall having received this piece of information because it lacks immediate salience.

Third, perhaps the 2008 study, which identified the gap in information about psychiatric disorders, was affected by selection bias-those parents who did not receive this kind 
of information were more likely to participate. ${ }^{14}$ Alternatively, perhaps medical geneticists who participated in the current study were those who are more cognizant of and likely to discuss psychiatric disorders related to 22q11DS. However, it is worth noting that our response rate was very respectable $(>50 \%)$ and that even among medical geneticists who participated in this study, it seems that there may in fact be a need for education about the psychiatric manifestations of 22q11DS, as illustrated by a quote from one participant:

"Cardiac defects and ca[lcium] defects can be fixed, immune def[iciency] can be managed, few disabilities can get early intervention. How helpless do you want to make parents feel about something we don't know the frequency of and that we don't know how to prevent?"

In fact, there is good information about the frequency with which individuals with 22q11DS develop psychiatric disorders. ${ }^{3}$ Moreover, as discussed above, although there is no prevention per se, early intervention for psychiatric disorders improves prognosis. Together, these suggest a possible role for continuing education for medical geneticists regarding psychiatric disorders in relation to 22q11DS; one participant expressed a sentiment to this effect in a comment on the survey:

"I became more aware of the psychiatric aspect (potential) of 22q deletion s[yndrome] after diagnosing this syndrome in a teenaged patient who presented with psychosis. I think there needs to be more education...."

Because medical geneticists who reported seeing the most patients with 22q11DS (10 or more cases per year) discussed psychiatric disorders more often than those who saw the fewest ( $0-1$ cases per year), it seems that awareness and adequate education about the disorder and the associated psychiatric problems may indeed play an important role in discussion practices.

A fourth potential explanation for the apparent potential discrepancy between the study by Hercher et al. ${ }^{14}$ and our current study is that, as with all studies using self-report surveys, social desirability is a potential limitation; therefore, it is possible that respondents do not actually discuss psychiatric manifestations of 22q11DS as frequently as they indicated.

\section{Limitations}

Potential limitations relating to ascertainment bias and social desirability that are common to self-report cross-sectional survey type studies have been discussed above. Other potential limitations relate to the fact that some of the respondents did not see patients from all four diagnostic scenarios (e.g., only seeing pediatric patients), limiting our ability to compare all responses across all time points. Because the OMS-HC stigma scale and the survey asked about psychiatric disorders in general, attitudes, opinion, and clinical practices relating to discrete diagnoses cannot be determined separately; this would be a potentially fruitful avenue of investigation for future work.

\section{Conclusion}

Our data show that medical geneticists discuss psychiatric manifestations of 22q11DS less frequently with families when the diagnosis is made in a child (perhaps due to the misperception that these conditions occur exclusively during adolescence/adulthood), and that frequency of discussion is associated with physicians' attitudes toward psychiatric disorders. Therefore, future studies might attempt to identify how, when, and by whom the psychiatric manifestations of the condition could be routinely brought to parents' attention to allow for timely treatment. Our data also suggest that implementing changes in training and continuing education for medical geneticists to reduce stigmatizing attitudes toward psychiatric disorders, ${ }^{23,29,38-40}$ and to increase awareness of the types and typical ages of onset of psychiatric conditions that people with 22q11DS can experience, have the potential to positively influence clinical practice.

\section{ACKNOWLEDGMENTS}

This study was funded by the Rare Disease Foundation, the Mitacs Accelerate Research Internship program, BC Mental Health and Addiction Services (BCMHAS), and the UBC Genetic Counseling program. The authors thank Maggie Hamidi, Anna Cantor, John Morris, and Patricia Birch for helping with various aspects of the study, and Boris Kuzeljevic for helping with the statistical analyses. J.A. was supported by the Canada Research Chairs Program, the Michael Smith Foundation for Health Research, and BCMHAS.

\section{DISCLOSURE}

The authors declare no conflict of interest.

\section{REFERENCES}

1. Devriendt K, Fryns JP, Mortier G, van Thienen MN, Keymolen K. The annual incidence of DiGeorge/velocardiofacial syndrome. J Med Genet 1998;35:789790.

2. Shprintzen RJ. Velo-cardio-facial syndrome: 30 Years of study. Dev Disabil Res Rev 2008;14:3-10.

3. Bassett AS, Chow EW, Husted J, et al. Clinical features of 78 adults with $22 q 11$ deletion syndrome. Am J Med Genet A 2005;138:307-313.

4. Green T, Gothelf D, Glaser B, et al. Psychiatric disorders and intellectual functioning throughout development in velocardiofacial (22q11.2 deletion) syndrome. J Am Acad Child Adolesc Psychiatry 2009;48:1060-1068.

5. Philip N, Bassett A. Cognitive, behavioural and psychiatric phenotype in 22q11.2 deletion syndrome. Behav Genet 2011;41:403-412.

6. Murphy KC, Jones LA, Owen MJ. High rates of schizophrenia in adults with velo-cardio-facial syndrome. Arch Gen Psychiatry 1999;56:940-945.

7. Niklasson L, Rasmussen P, Oskarsdóttir S, Gillberg C. Attention deficits in children with 22q.11 deletion syndrome. Dev Med Child Neurol 2005;47:803807.

8. Williams NM, Owen MJ. Genetic abnormalities of chromosome 22 and the development of psychosis. Curr Psychiatry Rep 2004;6:176-182.

9. Bassett AS, Hodgkinson K, Chow EW, Correia S, Scutt LE, Weksberg R. 22q11 deletion syndrome in adults with schizophrenia. Am J Med Genet 1998;81:328337.

10. Debbané M, Glaser B, David MK, Feinstein C, Eliez S. Psychotic symptoms in children and adolescents with 22q11.2 deletion syndrome: Neuropsychological and behavioral implications. Schizophr Res 2006;84:187-193.

11. Jolin EM, Weller RA, Weller EB. Psychosis in children with velocardiofacial syndrome (22q11.2 deletion syndrome). Curr Psychiatry Rep 2009;11:99-105.

12. Niklasson L, Rasmussen P, Oskarsdóttir S, Gillberg C. Autism, ADHD, mental retardation and behavior problems in 100 individuals with 22 q11 deletion syndrome. Res Dev Disabil 2009;30:763-773. 
13. Vorstman JA, Breetvelt EJ, Thode KI, Chow EW, Bassett AS. Expression of autism spectrum and schizophrenia in patients with a 22q11.2 deletion. Schizophr Res 2013;143:55-59.

14. Hercher L, Bruenner G. Living with a child at risk for psychotic illness: the experience of parents coping with 22q11 deletion syndrome: an exploratory study. Am J Med Genet A 2008;146A:2355-2360.

15. Martin N, Mikhaelian M, Cytrynbaum C, et al. 22q11.2 deletion syndrome: attitudes towards disclosing the risk of psychiatric illness. J Genet Couns 2012:21:825-834.

16. Bassett AS, McDonald-McGinn DM, Devriendt K, et al.; International 22q11.2 Deletion Syndrome Consortium. Practical guidelines for managing patients with 22q11.2 deletion syndrome. J Pediatr 2011;159:332-339.e1.

17. Young AS, Shashi V, Schoch K, Kwapil T, Hooper SR. Discordance in diagnoses and treatment of psychiatric disorders in children and adolescents with $22 q 11.2$ deletion syndrome. Asian J Psychiatr 2011;4:119-124.

18. Bales AM, Zaleski CA, McPherson EW. Patient and family experiences and opinions on adding 22q11 deletion syndrome to the newborn screen. J Genet Couns 2010;19:526-534.

19. Hallberg U, Oskarsdóttir S, Klingberg G. $22 q 11$ deletion syndrome - the meaning of a diagnosis. A qualitative study on parental perspectives. Child Care Health Dev 2010;36:719-725.

20. Corrigan PW, Watson AC. The stigma of psychiatric disorders and the gender, ethnicity, and education of the perceiver. Community Ment Health $\mathrm{J}$ 2007:43:439-458.

21. Kobau R, Diiorio C, Chapman D, Delvecchio P; SAMHSA/CDC Mental Illness Stigma Panel Members. Attitudes about mental illness and its treatment: validation of a generic scale for public health surveillance of mental illness associated stigma. Community Ment Health J 2010;46:164-176.

22. Brown SA. Factors and measurement of mental illness stigma: a psychometric examination of the Attribution Questionnaire. Psychiatr Rehabil J 2008;32: 89-94.

23. Chan JY, Mak WW, Law LS. Combining education and video-based contact to reduce stigma of mental illness: "the same or not the same" anti-stigma program for secondary schools in Hong Kong. Soc Sci Med 2009;68: $1521-1526$.

24. Keane M. Contemporary beliefs about mental illness among medical students: implications for education and practice. Acad Psychiatr 1990;3:172-177.

25. Watson AC, Corrigan PW, Ottati V. Police officers' attitudes toward and decisions about persons with mental illness. Psychiatr Serv 2004; 55:49-53.
26. Nordt C, Rössler W, Lauber C. Attitudes of mental health professionals toward people with schizophrenia and major depression. Schizophr Bull 2006;32: 709-714.

27. Schulze B. Stigma and mental health professionals: a review of the evidence on an intricate relationship. Int Rev Psychiatry 2007;19:137-155.

28. Monaco LC, Conway L, Valverde K, Austin JC. Exploring genetic counselors' perceptions of and attitudes towards schizophrenia. Public Health Genomics 2010;13:21-26

29. Anderson K, Austin JC. Effects of a documentary film on public stigma related to mental illness among genetic counselors. J Genet Couns 2012;21:573-581.

30. Church AH. Estimating the effect of incentives on mail survey response rates: a meta-analysis. Public Opinion Quarterly 1993;57:62-79.

31. Kassam A, Papish A, Modgill G, Patten S. The development and psychometric properties of a new scale to measure mental illness related stigma by health care providers: the Opening Minds Scale for Health Care Providers (OMS-HC). BMC Psychiatry 2012;12:62.

32. Sørensen KM, Agergaard $P$, Olesen $C$, et al. Detecting $22 q 11.2$ deletions by use of multiplex ligation-dependent probe amplification on DNA from neonatal dried blood spot samples. J Mol Diagn 2010;12:147-151.

33. Bertolote J, McGorry P. Early intervention and recovery for young people with early psychosis: consensus statement. Br J Psychiatry Supp/ 2005;48:s116-s119.

34. Onwumere J, Bebbington P, Kuipers E. Family interventions in early psychosis: specificity and effectiveness. Epidemiol Psychiatr Sci 2011;20:113-119.

35. Valmaggia LR, McGuire PK, Fusar-Poli P, Howes O, McCrone P. Economic impact of early detection and early intervention of psychosis. Curr Pharm Des 2012;18:592-595

36. Kohlbauer D, Meise $U$, Schenner M, et al. [Does education focusing on depression change the attitudes towards schizophrenia? A target-group oriented anti-stigma-intervention]. Neuropsychiatr 2010;24:132-140.

37. Reavley NJ, Jorm AF. Stigmatizing attitudes towards people with mental disorders: findings from an Australian National Survey of Mental Health Literacy and Stigma. Aust NZ J Psychiatry 2011;45:1086-1093.

38. Mann CE, Himelein MJ. Putting the person back into psychopathology: an intervention to reduce mental illness stigma in the classroom. Soc Psychiatry Psychiatr Epidemiol 2008;43:545-551.

39. Weiss MG, Ramakrishna J. Stigma interventions and research for international health. Lancet 2006;367:536-538

40. Corrigan PW, Larson J, Sells M, Niessen N, Watson AC. Will filmed presentations of education and contact diminish mental illness stigma? Community Ment Health J 2007:43:171-181. 